\title{
The Gaussian distribution of lattice size and atomic level heterogeneity in high entropy alloys
}

\author{
Shangyu Liu ${ }^{a}$, Yujie Wei ${ }^{\text {a,b,* }}$ \\ ${ }^{a}$ LNM, Institute of Mechanics, Chinese Academy of Sciences, Beijing 100190, China \\ ${ }^{\mathrm{b}}$ School of Engineering Sciences, University of Chinese Academy of Sciences, Beijing 100049, China
}

\section{G R A P H I C A L A B S T R A C T}
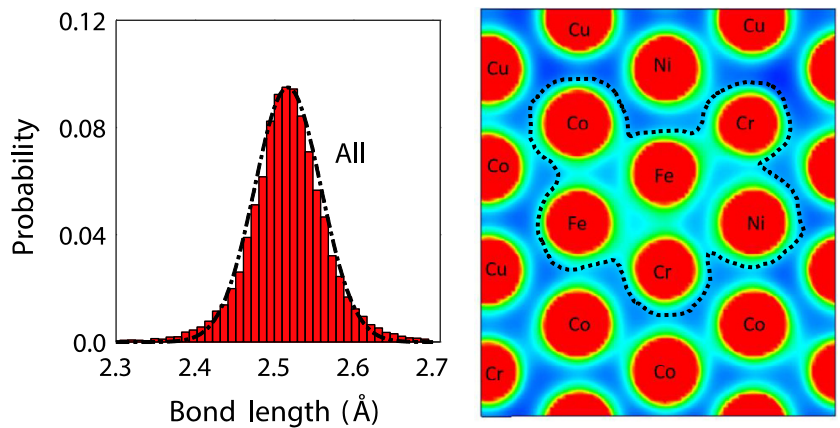

The bond length distribution of different pairs in high entropy alloy $\mathrm{FeCoNiCrCu}$ follows a Gaussian distribution (left). Such atomic heterogeneity is demonstrated via the charge density in FeCoNiCrCu: Atomic level clustering (surrounded by dotted lines) is clearly seen (right).

\section{A R T I C L E I N F O}

\section{Article history:}

Received 5 September 2016

Received in revised form

9 October 2016

Accepted 9 October 2016

Available online 19 October 2016

\section{Keywords:}

High entropy alloys

Gaussian distribution

Stacking fault energy

Bond length

Heterogeneity

\begin{abstract}
A B S T R A C T
It has been shown that heterogeneity at the micro-structure level can effectively enhance the strength and ductility of materials. Here we demonstrate via density-functional theory calculations that high entropy alloys (HEAs) exhibit atomic level heterogeneity in bond length and stacking fault energy (SFE) which could lead to HEAs' excellent hardenability and deformability. The bond length of HEA FeCoNiCrCu follows a Gaussian distribution, in contrast to the single-valued SFE and bond length in conventional crystalline counterparts. Its SFEs also fall into a wide span, which could introduce sequential and dispersed nucleation of different plastic sources including phase transformation, deformation twinning, and dislocations, giving rise to their exceptionally high hardenability and ductility. The atomic level heterogeneity in HEAs further gives rise to solid solution strengthening during dislocation motion. The concept of making atomic level heterogeneity can also be utilized in other metallic alloys for better hardenability and ductility.
\end{abstract}

(c) 2016 Elsevier Ltd. All rights reserved.

\footnotetext{
* Corresponding author at: LNM, Institute of Mechanics, Chinese Academy of Sciences, Beijing 100190, China.

E-mail address: yujie_wei@lnm.imech.ac.cn (Y. Wei).
}

\section{Introduction}

Among possible routines to evade strength-ductility tradeoff in metallic materials, [1-3] microstructure heterogenization is broadly employed. For example, tuning grains with bi-modal 
size distribution in crystalline metals is capable of increasing their tensile ductility of crystalline metals [4]. Multi-phase alloys, such as dual-phase steels, are another type of model case to demonstrate the effectiveness of microstructure heterogenization for better strength and ductility $[3,5,6]$. By adding dispersed crystalline phase in monolithic metallic glasses, researchers found the fracture toughness could be significantly increased [6]. More recently, heterogeneous microstructures with gradient has also been explored $[2,7,8]$, which show a synergetic effect in realizing better strength and ductility. In parallel to synthesize materials with heterogeneous microstructures, there is a long-standing trend of adding some fraction of dissolution atoms in metals to improve their performance. This strategy, as we will demonstrate via HEAs, is essentially to tune the intrinsic properties to be heterogeneous.

Unlike conventional alloys, HEAs are composed of multiple principal elements with nearly equal concentrations [9]. The mixture of principal elements leads to server lattice distortion thereby giving high strength and exceptional performance at elevated temperature [9-12]. Due to the random distribution of atoms of different size, the interfacial properties of HEAs are heterogeneous, in contrast to their conventional single valued counterparts. One of the most significant parameters governs the plastic deformation mechanism is the stacking fault energy: (1) Stacking fault energy influences the competition between partial dislocation nucleation and full dislocation as [13] the critical resolved shear stress for dislocation nucleation is proportional to the SFE [14]. Combining with grain size or twin spacing, SFE plays a central role in determining the strength of nanostructured materials [14-17]. (2) Stacking fault energy is associated with brittle-ductile transition in crystalline materials $[18,19]$ as the nucleation of dislocations versus twins is related to crack tip blunting or cleavage. (3) Stacking fault energy relates to phase transformation. It has been generally recognized that low intrinsic stacking fault energy $\gamma_{s f}$ favors the $\gamma$ (face-centered cubic, FCC phase) to $\varepsilon$ (hexagonal close-packed, HCP phase) transformation [20,21]. Because of the alloying of atoms of different size in HEAs, the lattice size varies correspondingly, which leads to the variation in SFEs as well. Hence it is desired to have a comprehensive understanding about the lattice size distribution of HEAs and SFEs, which could be the origin of their superb mechanical properties. Since HEAs are primarily composed of single phase like FCC or body-centered cubic (BCC) other than intermetallic compounds, we investigate the SFE of HEAs in a relatively traditional way [22]. We examine how the huge disorder in atomic scale in HEAs leads to a distributed SFE other than a converged value in pure metals. In addition, we explore other atomic level heterogeneity like bond structure and charge-density distribution.

\section{Calculation details}

All our calculations were fulfilled by using the Vienna ab initio simulation package (VASP) $[23,24]$ based on density functional theory (DFT). Projector augmented wave (PAW) pseudopotentials and the Perdew-Wang 1991 [25] chosen for exchange and correlation interaction in the generalized gradient approximation (GGA) were used in VASP calculations. The FCC high entropy alloys' models were illustrated in Fig. 3(a): The supercell containing 6layers stacking along $\{111\}$ plane, and there are 16 atoms in each layer. In practical calculations, we employed a supercell with 12 layers. The lengths of $x$-, $y$ - and $z$-direction in one supercell box are about $8.5 \AA$, 10 Åand $12 \AA$, respectively. The Brillouin zone is sampled by a $4 \times 4 \times 3$ Monkhorst and Pack grid for all calculations of HEAs. And the cutoff energy was set to $400 \mathrm{ev}$. We relaxed all the similar supercells' total energy to the precision of $0.01 \mathrm{meV}$. The distributions of different atom-pairs for both the first nearest neighbors and the second nearest neighbors are then extracted from the equilibrated samples.
The intrinsic stacking fault energy we calculated is defined as $E_{\text {isf }}=\left(E_{\text {fault }}-E_{\text {perfect }}\right) / A$, where $E_{\text {fault }}$ and $E_{\text {perfect }}$ are the free energy of the perfect structure and the structure contained onelayer fault, respectively, and $A$ is the area of one layer. To validate the effectiveness of the method, we firstly did a benchmark test and calculated the SFE of pure copper. The SFE of Cu along the $\{111\}$ plane is $39 \mathrm{~mJ} / \mathrm{m}^{2}$, in good agreement with the value reported in literature $\left(41 \mathrm{~mJ} / \mathrm{m}^{2},[26]\right)$. Now we start to investigate the SFE of HEA FeCoNiCrCu, which is believed to be FCC phase at room temperature $[27,28]$. It has 5 types of atoms and the numbers of each type were set as 38, 39, 38, 38, and 39 for $\mathrm{Fe}, \mathrm{Co}, \mathrm{Ni}$, $\mathrm{Cr}, \mathrm{Cu}$, in turn. We specify atom types at different positions with random numbers and generated 10 random models. To obtain the structure with one layer fault, we produced a rigid displacement between two adjacent layers, and the magnitude equals to that of the Burgers vector $b_{p}=1 / 6\langle 112\rangle$, as illustrated in Fig. 3(a) and (b).

\section{Results and discussion}

\subsection{The Gaussian distribution of lattice size}

The distributed SFE in HEAs ought to be connected with the structure of atoms. To shed light on this, we analyze bond lengths of the HEAs at equilibrium. The averaged bond lengths of different atom-pairs in the two systems are summarized in Fig. 1. Fig. 1(a)-(c) show the distributions of bond lengths of the first nearest neighbors of the types of atom-pair $\mathrm{Fe}-\mathrm{Fe}, \mathrm{Fe}-\mathrm{Cr}$, $\mathrm{Cr}-\mathrm{Cr}$, respectively. If counting the bond length of the first nearest neighbors, it follows the Gaussian distribution

$$
g(x)=\frac{1}{\sqrt{2 \pi \delta^{2}}} e^{-\frac{(x-\Lambda)^{2}}{2 \delta^{2}}},
$$

where $x$ represents the bond length variable, $\Lambda$ and $\delta^{2}$ are the mean and the variance of the bond length distribution. We see in Fig. 1(d) that a mean of $\Lambda=2.517 \AA$ and a variance of $\delta^{2}=0.00176 \AA^{2}$ could match the DFT results well. The bond length distributions of the second nearest neighbors of atom-pair types $\mathrm{Fe}-\mathrm{Fe}, \mathrm{Fe}-\mathrm{Cr}$, $\mathrm{Cr}-\mathrm{Cr}$ are shown in Fig. 1(e)-(g), respectively, and the bondlength distribution of all the second nearest neighbors is shown in Fig. 1(g). Bond lengths of the second nearest neighbors also follow Gaussian distribution, with $\Lambda=3.558 \AA$ and $\delta^{2}=0.003 \AA^{2}$. In Table 1, we list the corresponding mean bond length, the variance, and the standard deviation of bond length for different atom pairs. It seems that the scattering of different bond types could be well represented by the Gaussian distribution.

The fluctuation in bond length due to element mixture in HEAs could be well demonstrated from the radial distribution function. We show in Fig. 2 the radial distribution of $\mathrm{FeCoNiCrCu}$ and other typical metals with different structures. As well known, atoms in regular crystalline materials like face-centered cubic metals are ordered. Their neighboring atoms could be found at characteristic distances, which lead to single-valued peaks in the radial distribution function seen in Fig. 2(b) and (c) for FCC iron and BCC iron, respectively. For atoms in HEAs, while they are also regarded as ordered and dense, their spacing is not single-valued and falls in a distribution. Consequently, their neighboring atoms are placed with an averaged characteristic distance which falls in a certain range. Such features give rise to a smooth distribution at each characteristic distance seen in Fig. 2(a). For highly disordered and usually less dense materials like metallic glasses, atoms are placed irregularly. We could essentially find their neighbors at any approximate distances. This nature leads to a smooth variation in radial distribution, as shown in Fig. 2(d).

\subsection{Stacking fault energy of high entropy alloys}

The stacking fault energies of $\mathrm{FeCoNiCrCu}$ from 52 independent calculations are shown in Fig. 3(c). It is seen that SFE values of these 

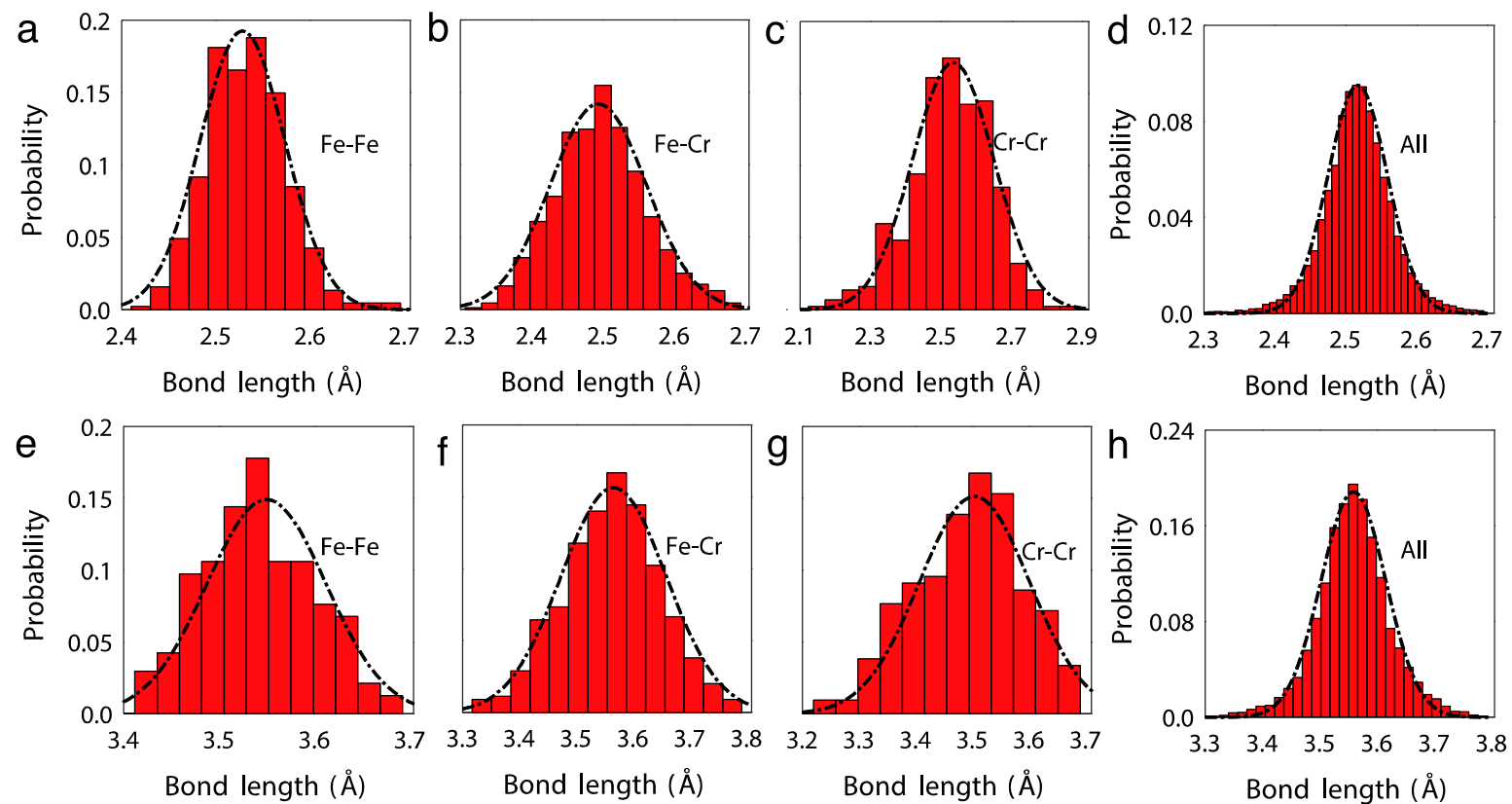

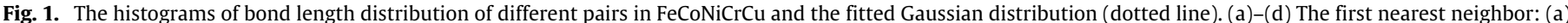
Fe-Fe pair, (b) Fe-Cr pair, (c) Cr-Cr Pair, (d) All bond pairs. (e)-(h) The second nearest neighbor: (e) Fe-Fe pair, (f) Fe-Cr pair, (g) Cr-Cr Pair, (h) All bond pairs.
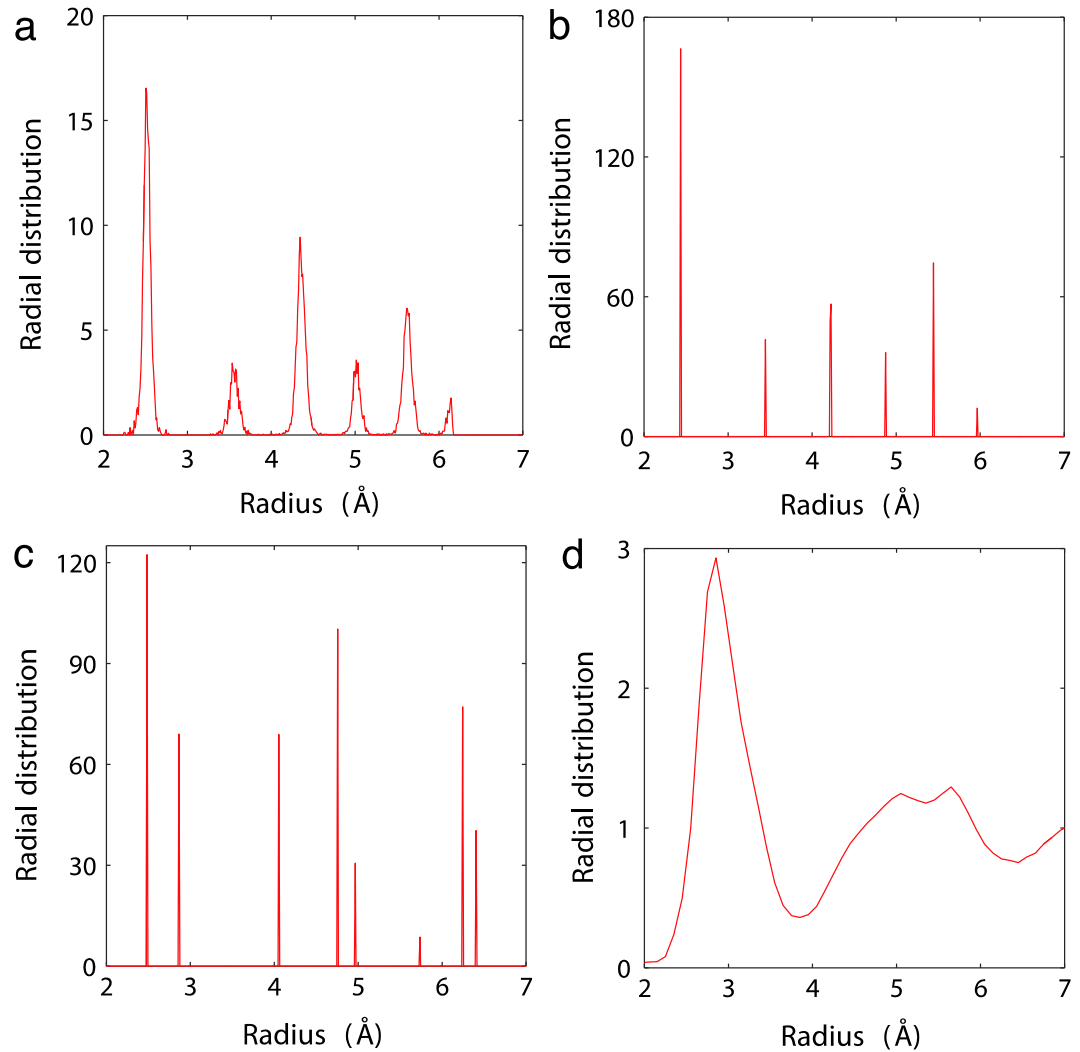

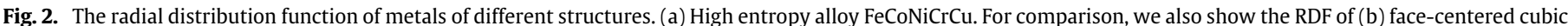
Fe and (c) body-centered cubic Fe (c), as well as RDF of (d) a binary metallic glass Cu50Zr50 based on molecular dynamics model with 50000 Atoms.

Table 1

The mean $(\Lambda)$, the variance $\left(\delta^{2}\right)$, and the standard deviation (STD) of the bond length of different atom pairs.

\begin{tabular}{|c|c|c|c|c|c|c|c|c|}
\hline & \multicolumn{3}{|c|}{ The first nearest neighbor } & \multicolumn{5}{|c|}{ The second nearest neighbor } \\
\hline & All & $\mathrm{Cr}-\mathrm{Cr}$ & $\mathrm{Fe}-\mathrm{Cr}$ & $\mathrm{Fe}-\mathrm{Fe}$ & All & $\mathrm{Cr}-\mathrm{Cr}$ & $\mathrm{Fe}-\mathrm{Cr}$ & $\mathrm{Fe}-\mathrm{Fe}$ \\
\hline$\Lambda,(\AA)$ & 2.5170 & 2.5096 & 2.4933 & 2.5178 & 3.5585 & 3.5159 & 3.5609 & 3.5475 \\
\hline$\delta^{2}\left(\AA^{2}\right)$ & 0.00176 & 0.01197 & 0.00436 & 0.00171 & 0.00319 & 0.01027 & 0.00829 & 0.00380 \\
\hline STD & 0.042 & 0.1094 & 0.0661 & 0.0414 & 0.0565 & 0.1014 & 0.0911 & 0.0617 \\
\hline
\end{tabular}



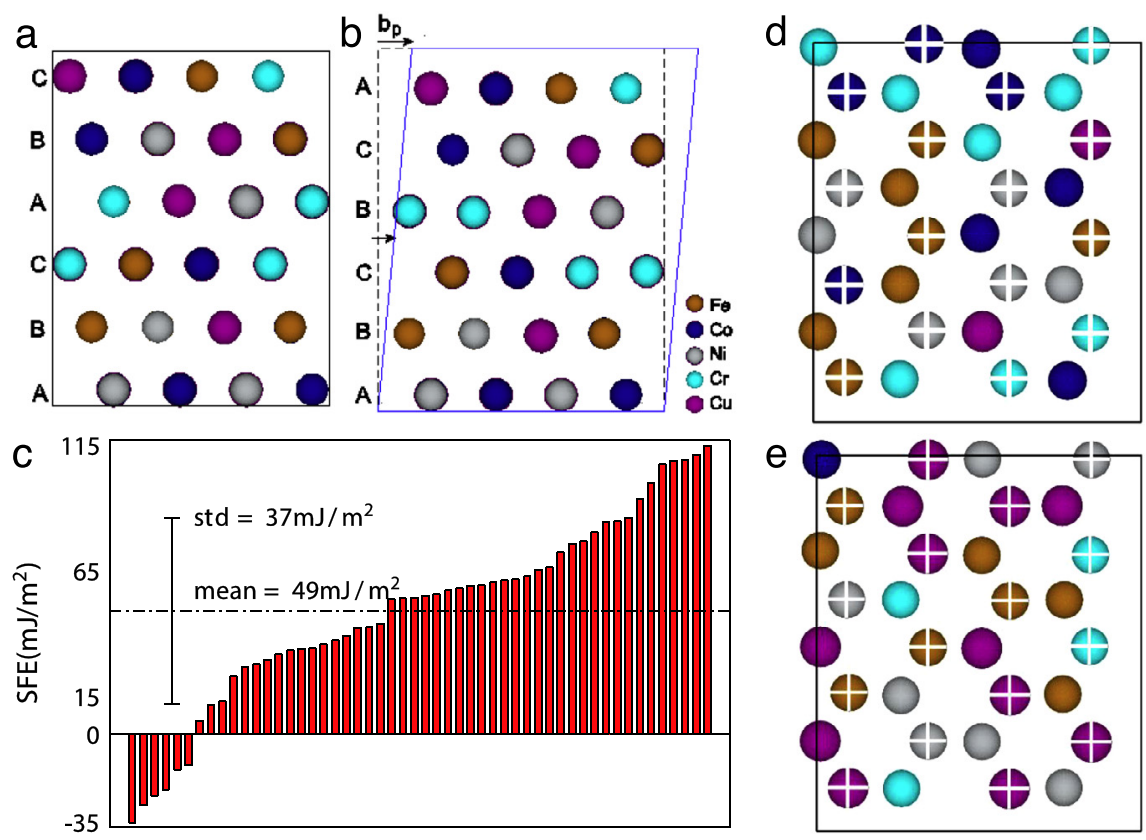

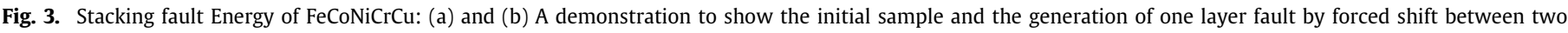

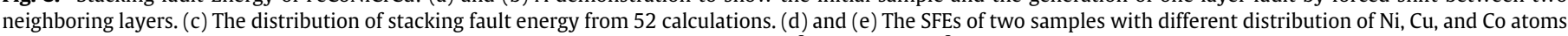

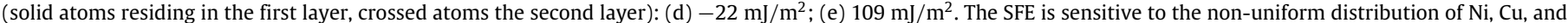
Co atoms: more Ni and $\mathrm{Cu}$ for higher SFEs and more Co for lower SFEs.

samples distribute in a wide range and could even be negative. This distribution of SFEs seems to be strongly correlated with the non-uniform distribution of $\mathrm{Ni}, \mathrm{Cu}$ and $\mathrm{Co}$ atoms, as illustrated in Fig. 3(d) and (g). The SFEs between two atomic planes with more $\mathrm{Ni}$ and $\mathrm{Cu}$ (Fig. 3(e)) are much higher than those with less $\mathrm{Ni}$ or $\mathrm{Cu}$ atoms (Fig. 3(d)); in contrast, less Co atoms lead to higher SFEs. Since the SFE at atomic scale in HEAs is not single valued, the distribution of SFE may introduce the activation of different plastic mechanisms. This feature is of great meaning for the strength and ductility in these high entropy alloys.

Indeed, the significance of SFE in tuning the properties of crystalline materials has been exemplified in the tunable performance of Hadfield steels. It has been demonstrated that through adjusting the atomic fraction of manganese of Fe-Mn austenitic steels [20,21,29], one may control the activation of different types of plastic sources. The essence is to use manganese to manipulate the SFE $[20,29]$. For FCC crystals of low SFE, say approximately $<20 \mathrm{~mJ} / \mathrm{m}^{2}$, plasticity is primarily achieved by the $\gamma$ (FCC phase) to $\varepsilon$ (HCP phase) transformation, accompanied with partial dislocation gliding. When SFE falls within the range of $20-420 \mathrm{~mJ} / \mathrm{m}^{2}$, deformation twinning is dominant, in particular at the early stage of plastic deformation [21]. For SFE higher than $420 \mathrm{~mJ} / \mathrm{m}^{2}$, full dislocations gliding plays the leading role. Deformation twinning is known to promote tensile ductility and gives rise to enormous strain hardening, while phase transformation helps for high yield strength with intermediate hardening rate. The combined effects lead to the superb hardenability and ductility in high manganese steels. Given the similarity between FCC HEAs with Hadfield steels, the distribution of SFE helps to realize the activation of several plastic deformation mechanisms in one HEA, in contrast to one primary plastic deformation in a Hadfield steel with a given manganese fraction. The activation of multiple plastic deformation mechanisms in HEAs could hence result in an excellent combination of hardening rate, ultimate strength and tensile ductility.

\subsection{Atomic level heterogeneous clustering}

In addition to the bond structures, we also extract the charge density in the HEA from our density functional theory calculations.
Fig. 4(a)-(b) show the charge density in $\mathrm{FeCoNiCrCu}$ in two representative regions. In addition to the bond length distribution, there is a trend to form tightly bound clusters: The pattern in Fig. 4(a) shows a cluster composed of $\mathrm{Co}-\mathrm{Cr}-\mathrm{Fe}-\mathrm{Ni}$ atoms. Atoms in the $\mathrm{Co}-\mathrm{Cr}-\mathrm{Ni}$ cluster in Fig. 4(b) seem to be closer than other pairs in terms of charge density. The information extracted from the charge density in HEAs conforms to the corresponding distribution of bonds length. In addition to the bond-level heterogeneity, the clusters composed of multiple atoms could promote heterogeneous plastic deformation mechanisms, which is beneficial to the strengthening and toughening of HEAs.

\section{Conclusion and discussion}

It has been broadly accepted that stacking fault energy is a governing parameter for plasticity in crystalline materials, in particular at the nucleation stage of dislocations [13-15]. In this work, we systematically investigate the stacking fault energy of the typical HEA FeCoNiCrCu. It is found that the SFEs in the HEA are highly fluctuating and statistical in nature, in contrast to single valued SFEs in their crystalline counterparts. Since the SFE influences the selection of primary plastic deformation mechanisms and subsequent interactions of substructures resulted from plastic deformation, it plays a critical role for the strength and ductility of alloys. Traditionally, we promote strain hardening by introducing heterogeneity in texture distribution, grain size distribution, substructures like dislocation cells and deformation twins. The enormous strain hardenability in HEAs is a combination of hardening mechanisms with heterogeneous stacking fault energies. The scattering in SFE may supply multifold hardening mechanisms: (1) Dislocation nucleation will gradually shift from interfaces with lower $\gamma_{i s f}$ to higher ones as deformation proceeds, as the critical resistance for partial dislocation nucleation is $\tau_{c}=\frac{\gamma_{\text {isf }}}{b}+\frac{G b}{3 d}$ [14]. (2) The primary plastic deformation mechanism may switch as $\gamma_{\text {isf }}$ influences the competition among phase transformation, partial dislocation gliding, deformation twinning, and complete dislocation motion $[20,21]$. Increasing deformation will lead to the depletion of plastic deformation in regions with low SFEs. Higher stress is then required in order to trigger possibly different plasticity mechanisms 

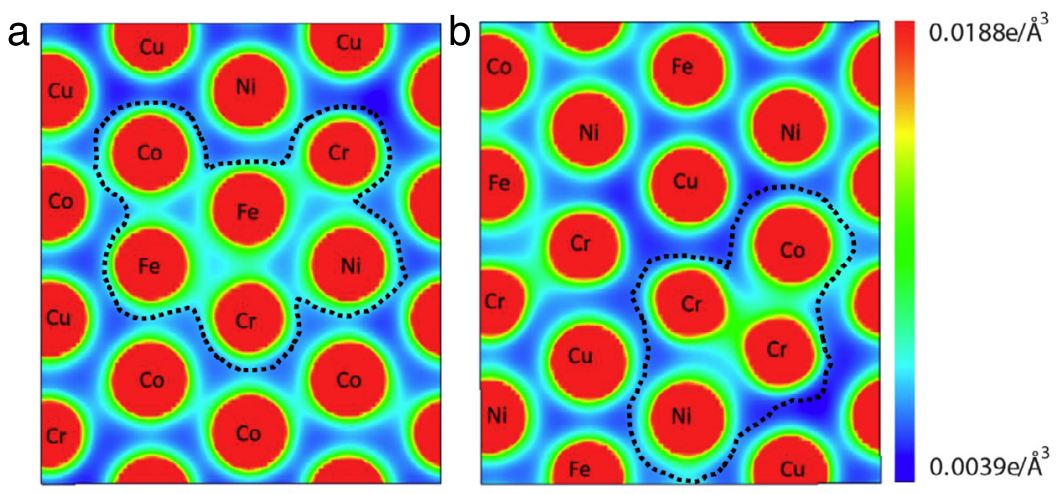

Fig. 4. Cross-sectional view to show charge density in high entropy alloy FeCoNiCrCu: (a) and (b) The charge density of two representative regions in FeCoNiCrCu to show the clustering (surrounded by dotted lines) at atomistic level.

in regions of higher SFEs, which leads to the switch of primary plastic deformation mechanisms. (3) The newly formed yet highly dispersed phase boundary, stacking faults, twin boundaries, and dislocation walls resulted from the distribution of SFEs would then supply additional hardening as deformation continues [14,16]. In view of those, it is natural to see that heterogeneous SFE in HEAs would give rise to extraordinary mechanical properties, like damage tolerance at nanoscale [30]. The fluctuation in SFEs in HEAs originates from the mixing of atoms of different size: That was further verified by investigation on the distribution of lattice size of the same atompair in the HEA system shown in Fig. 1 and its radial distribution function presented in Fig. 2. The lattice distortion resulted from bond-length fluctuation gives rise to solid solution strengthening mechanism. The atomic scale heterogeneity due to non-uniform bond lengths in HEAs is equivalent to the local modulus mismatch and size mismatch between solvent and solute atoms. They both generate nanoscale heterogeneous stress field which introduces additional resistance to gliding dislocations. The distribution nature of SFE and lattice structures in HEAs revealed here paves the way for an alternative design strategy to improve the strength and ductility of crystalline metals by introducing intrinsic and atomic level heterogeneity. Such features could be also used as input for constitutive models $[31,32]$ to capture the mechanical and deformation behavior of HEAs.

\section{Acknowledgments}

The authors acknowledge support from the National Natural Science Foundation of China (Grant no. 11425211).

\section{References}

[1] R.O. Ritchie, The conflicts between strength and toughness, Nature Mater. 10 (11) (2011) 817-822

[2] Y. Wei, Y. Li, L. Zhu, Y. Liu, X. Lei, G. Wang, Y. Wu, Z. Mi, J. Liu, H. Wang, Evading the strength-ductility trade-off dilemma in steel through gradient hierarchical nanotwins, Nature Commun. 5 (4) (2014) 3580.

[3] Z. Li, K.G. Pradeep, Y. Deng, D. Raabe, C.C. Tasan, Metastable high-entropy dualphase alloys overcome the strength-ductility trade-off, Nature 534 (2016) $227-230$.

[4] E. Ma, E. Ma, Eight routes to improve the tensile ductility of bulk nanostructured metals and alloys. Jom-Us 58, 49-53, JOM: J. Miner. Met Mater. Soc. 58 (4) (2006) 49-53.

[5] M. Delincé, Y. Bréchet, J.D. Embury, M.G.D. Geers, P.J. Jacques, T. Pardoen, Structure-property optimization of ultrafine-grained dual-phase steels using a microstructure-based strain hardening model, Acta Mater. 55 (7) (2007) 2337-2350.

[6] D.C. Hofmann, J.-Y. Suh, A. Wiest, G. Duan, M.-L. Lind, M.D. Demetriou, W.L. Johnson, Designing metallic glass matrix composites with high toughness and tensile ductility, Nature 451 (7182) (2008) 1085-1089.

[7] T.H. Fang, W.L. Li, N.R. Tao, K. Lu, Revealing extraordinary intrinsic tensile plasticity in gradient nano-grained copper, Science 331 (6024) (2011) 1587-1590.

[8] X. Wu, P. Jiang, L. Chen, F. Yuan, Y.T. Zhu, Extraordinary strain hardening by gradient structure, Proc. Natl. Acad. Sci. 111 (20) (2014) 7197-7201.
[9] Y. Zhang, T.T. Zuo, Z. Tang, M.C. Gao, K.A. Dahmen, P.K. Liaw, Z.P. Lu, Microstructures and properties of high-entropy alloys, Prog. Mater. Sci. 61 (2014) 1-93.

[10] Y. Deng, C.C. Tasan, K.G. Pradeep, H. Springer, A. Kostka, D. Raabe, Design of a twinning-induced plasticity high entropy alloy, Acta Mater. 94 (2015) $124-133$.

[11] B. Gludovatz, A. Hohenwarter, D. Catoor, E.H. Chang, E.P. George, R.O. Ritchie A fracture-resistant high-entropy alloy for cryogenic applications, Science 345 (6201) (2014) 1153-1158.

[12] M.J. Yao, K.G. Pradeep, C.C. Tasan, D. Raabe, A novel, single phase, nonequiatomic FeMnNiCoCr high-entropy alloy with exceptional phase stability and tensile ductility, Scr. Mater. 72-73 (2014) 5-8.

[13] J.P.H.J. Lothe, Theory of Dislocations, second ed., 1982.

[14] R.J. Asaro, S. Suresh, Mechanistic models for the activation volume and rate sensitivity in metals with nanocrystalline grains and nano-scale twins, Acta Mater. 53 (12) (2005) 3369-3382.

[15] K. Lu, L. Lu, S. Suresh, Strengthening materials by engineering coherent internal boundaries at the nanoscale, Science 324 (5925) (2009) 349-352.

[16] T. Zhu, H. Gao, Plastic deformation mechanism in nanotwinned metals: An insight from molecular dynamics and mechanistic modeling, Scr. Mater. 66 (11) (2012) 843-848.

[17] Y. Wei, Scaling of maximum strength with grain size in nanotwinned fcc metals, Phys. Rev. B 83 (13) (2011) -

[18] J.R. Rice, Dislocation nucleation from a crack tip: An analysis based on the Peierls concept, J. Mech. Phys. Solids 40 (2) (1992) 239-271.

[19] E.B. Tadmor, S. Hai, A Peierls criterion for the onset of deformation twinning at a crack tip, J. Mech. Phys. Solids 51 (51) (2003) 765-793.

[20] K. Sato, M. Ichinose, Y. Hirotsu, Y. Inoue, K. Sato, M. Ichinose, Effects of deformation induced phase transformation and twinning on the mechanical properties of austenitic Fe-Mn-Al alloys, Isij Int. 52 (29) (2010) 868-877.

[21] Y. Li, L. Zhu, Y. Liu, Y. Wei, Y. Wu, D. Tang, Z. Mi, On the strain hardening and texture evolution in high manganese steels: Experiments and numerical investigation, J. Mech. Phys. Solids 61 (12) (2013) 2588-2604.

[22] A.J. Zaddach, C. Niu, C.C. Koch, D.L. Irving, Mechanical properties and stacking fault energies of NiFeCrCoMn high-entropy alloy, Jom J. Miner. Met. Mater. Soc. 65 (12) (2013) 1780-1789.

[23] G. Kresse, J. Hafner, Ab initio molecular dynamics for liquid metals, Phys. Rev. B 47 (1) (1993) 558.

[24] G. Kresse, J. Furthmüller, Efficient iterative schemes for ab initio total-energy calculations using a plane-wave basis set, Phys. Rev. B 54 (16) (1996) 11169.

[25] J.P. Perdew, P. Ziesche, H. Eschrig, Electronic Structure of Solidś, Vol. 91, Akademie Verlag, Berlin, 1991.

[26] S. Kibey, J.B. Liu, D.D. Johnson, H. Sehitoglu, Predicting twinning stress in fcc metals: Linking twin-energy pathways to twin nucleation, Acta Mater. 55 (20) (2007) 6843-6851.

[27] H.B. Cui, L.F. Zheng, J.Y. Wang, Microstructure evolution and corrosion behavior of directionally solidified FeCoNiCrCu high entropy alloy, Appl. Mech. Mater. 66-68 (3) (2011) 146-149.

[28] F. Otto, Y. Yang, H. Bei, E.P. George, Relative effects of enthalpy and entropy on the phase stability of equiatomic high-entropy alloys, Acta Mater. 61 (7) (2013) 2628-2638.

[29] S. Allain, J.P. Chateau, O. Bouaziz, S. Migot, N. Guelton, Correlations between the calculated stacking fault energy and the plasticity mechanisms in Fe-Mn-C alloys, Mater. Sci. Eng. A 387-389 (1) (2004) 158-162.

[30] Z.J. Zhang, M.M. Mao, J. Wang, B. Gludovatz, Z. Zhang, S.X. Mao, E.P. George, O $\mathrm{Yu}, \mathrm{R} . \mathrm{O}$. Ritchie, Nanoscale origins of the damage tolerance of the high-entropy alloy CrMnFeCoNi, Nature Commun. 6 (2015).

[31] Z. Wu, Y.F. Gao, H. Bei, Single crystal plastic behavior of a single-phase, face-center-cubic-structured, equiatomic FeNiCrCo alloy $\hat{\omega}^{2}$, Scr. Mater. 109 (2015) 108-112.

[32] Z. Wu, Y. Gao, H. Bei, Thermal activation mechanisms and Labusch-type strengthening analysis for a family of high-entropy and equiatomic solidsolution alloys, Acta Mater. 120 (2016) 108-119. 